MINIREVIEW

\title{
WNK1: analysis of protein kinase structure, downstream targets, and potential roles in hypertension
}

\author{
Bing-e XU ${ }^{1}$, Byung-Hoon LEE ${ }^{1}$, Xiaoshan MIN ${ }^{2}$, Lisa LENERTZ ${ }^{1}$, Charles J HEISE ${ }^{1}$, Steve STIPPEC ${ }^{1}$, Elizabeth J \\ GOLDSMITH $^{2}$, Melanie H. COBB ${ }^{1, *}$ \\ ${ }^{I}$ Department of Pharmacology, University of Texas Southwestern Medical Center, 5323 Harry Hines Blvd, Dallas, USA. \\ ${ }^{2}$ Department of Biochemistry, University of Texas Southwestern Medical Center, 5323 Harry Hines Blvd, Dallas, USA.
}

\begin{abstract}
The WNK kinases are a recently discovered family of serine-threonine kinases that have been shown to play an essential role in the regulation of electrolyte homeostasis. Intronic deletions in the WNK1 gene result in its overexpression and lead to pseudohypoaldosteronism type II, a disease with salt-sensitive hypertension and hyperkalemia. This review focuses on the recent evidence elucidating the structure of the kinase domain of WNK1 and functions of these kinases in normal and disease physiology. Their functions have implications for understanding the biochemical mechanism that could lead to the retention or insertion of proteins in the plasma membrane. The WNK kinases may be able to influence ion homeostasis through its effects on synaptotagmin function.
\end{abstract}

Keywords: kinase, hypertension, synaptotagmin, MAP3K, MAP4K, ERK5, RNAi.

\section{INTRODUCTION}

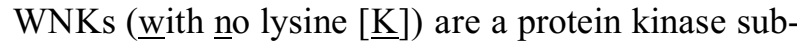
family with a unique placement of the lysine that is involved in binding ATP and catalyzing phosphoryl transfer [1]. WNKs occur in multicellular organisms, but WNK homologs are not present in unicellular organisms such as $S$. cerevisiae [1-6]. Several functions of WNKs have now been identified, but additional clues may come from Arabidopsis thaliana which has the largest number of WNK family members known, more than twice the number in human. Interestingly, some of these WNKs are implicated in controlling circadian rhythms [3, 4].

Two WNK family members have been associated with a rare form of monogenic hypertension known as pseudohypoaldosteronism type II (PHA II) [6]. Lifton and colleagues made this determination by positional cloning, defining mutations in genes encoding WNK1 and WNK4 in affected members of families with a high incidence of PHA II. While the mutations in WNK4 are in the coding sequence causing point mutations, the mutations in WNK1 occur due to large deletions in the first intron, with no changes in coding sequence. The consequence of the

*Correspondence: Melanie H. COBB

Tel: 214-648-3627, Fax: 214-648-3811,

E-mail: melanie.cobb@UTSouthwestern.edu intronic mutations is believed to be an increase of up to five-fold in WNK1 expression. Disruption of the WNK1 gene in mice leads to embryonic lethality before embryonic day 13 [7]. Heterozygotes survive but display reduced blood pressure, consistent with the role of WNK1 in regulation of blood pressure in human. Because we originally isolated a cDNA encoding WNK1, we have concentrated on characterizing its properties and identifying WNK1-dependent mechanisms that could lead to hypertension.

\section{STRUCTURAL ANALYSIS OF WNK1}

When we originally examined the rat WNK1 sequence, we found that it contained a kinase domain near its N-terminus and a long $\mathrm{C}$-terminus with predicted coiled coils, but no other identifiable domains (Fig. 1A) [1]. In addition to its large size, $\sim 230 \mathrm{kD}$, its most surprising feature was the absence of the lysine normally present in $\beta$ strand 3 , subdomain II, of the protein kinases, that was thought to be invariant in the family. Through a structure/function analysis we found the position of the catalytic lysine in $\beta$ strand 2, a part of the glycine loop. A comparison of all of the WNK sequences shows conservation of the lysine in that position. Crystal structure analysis supports the conclusion that the catalytic lysine is derived from $\beta$ strand 2 not $\beta$ strand 3 , the position in all other protein kinases (Fig. 1B) [8]. Analysis of the protein kinase activities of a series of 
A

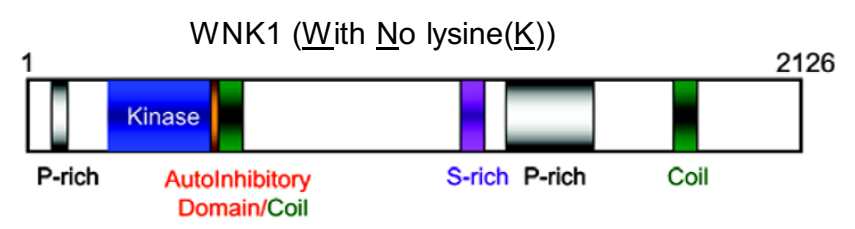

B

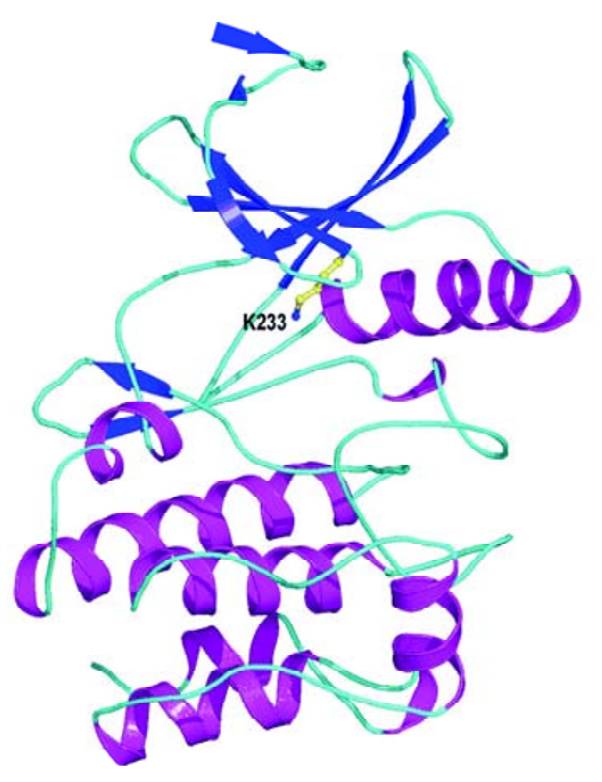

Fig. 1 WNK1 has a unique structure. (A) Schematic of WNK1 structure. (B) Crystal structure of the catalytic domain of WNK1 with serine 382 mutated to alanine and lysine 233 indicated.

WNK1 truncation mutants also revealed the presence of an autoinhibitory domain and an obligate autophosphorylation site in the activation loop [9].

\section{WNK1 IS UBIQUITOUS}

We used immunoblotting to determine the pattern of WNK1 expression and found that it is ubiquitously expressed in tissues and cell lines. For example, it is readily detected in cell lines derived from a variety of tissues (Fig. 2A). Its amount per unit protein in distal convoluted tubule cells (DCT) was among the lowest we found. This is probably due to the fact that WNK1 has multiple splice forms; and kidney primarily expresses a form that lacks 384 residues from its $\mathrm{N}$-terminus, yielding a smaller, noncatalytic protein that would not have been detected with our antibodies. Among a range of agents tested, $\mathrm{NaCl}$ and other osmotic stresses are the most clear cut WNK1 activators (Fig. 2B). A number of hormones and other types of stress, such as heat shock, have little effect on WNK1 catalytic activity.
A

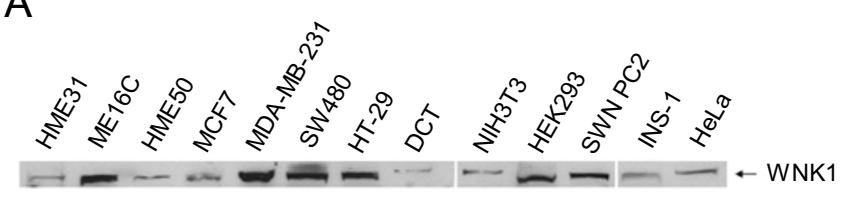

B

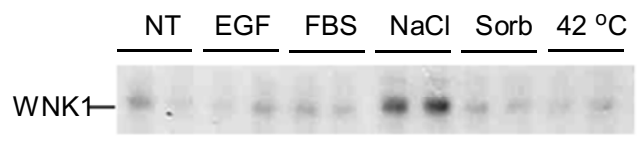

Fig. 2 WNK1 is ubiquitously expressed. (A) Immunoblot of protein from soluble lysates of the indicated cell lines. (B) Activation of WNK1 from COS cells by extracellular agents.

\section{WNK1 HAS MAP4K ACTIVITY IN THE ERK5 PATHWAYS}

Because WNK1 is a distant relative of Ste20p, an enzyme upstream in the MAP kinase (MAPK) cascade in the yeast mating pathway, we examined the potential role of WNK1 in activating MAPK cascades in mammalian cells. MAPK cascades are pleiotropic regulatory pathways that assist in the control and coordination of numerous cellular processes including the cell cycle, transcription, apoptosis and proliferation $[10,11]$. MAPK cascades consist of a MAPK activated by a MAPK kinase (MAP2K or MEK) which must also be activated by an upstream MAPK kinase kinase (MAP3K) (Fig. 3A). MAP3Ks are often also regulated by MAP4Ks as well as by association with scaffolds or adapters.

We found that WNK1 had no detectable capacity to activate either the ERK $1 / 2$ or the JNK MAPK pathways, and only a weak, marginal effect on p38 MAPK. However, we found that overexpressed WNK1 clearly activated ERK5 (Fig. 3B). In contrast to most members of the MAPK family, ERK5 has a long C-terminal tail which has been suggested to have a variety of functions [12]. The kinase domain of ERK 5 is most similar to ERK2. ERK5 is activated by growth factors such as epidermal growth factor (EGF), as well as by osmotic and other types of stress including sorbitol, $\mathrm{H}_{2} \mathrm{O}_{2}$, and UV irradiation [13, 14]. The ERK5 MAPK pathway contains the MAP2K MEK5, and the MAP3Ks MEKK2 and MEKK3 as the upstream activators of ERK5 [15, 16]. Like other MAPKs, ERK5 has several transcription factor substrates including the MEF2 family members, MEF2A, C and D, and the ETS-like transcription factor SAP1a [17, 18]. ERK5 has been implicated in neuronal survival and cell proliferation $[18,19]$.

MEKK2 and MEKK3 are closely related MAP3Ks that have been shown to regulate the $\mathrm{p} 38$, JNK, and the ERK5 MAPK cascades. As with other MAP3Ks, both bind to 
A

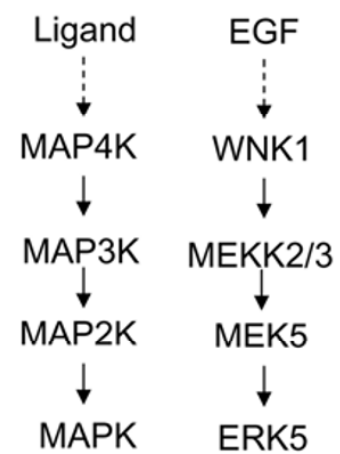

B

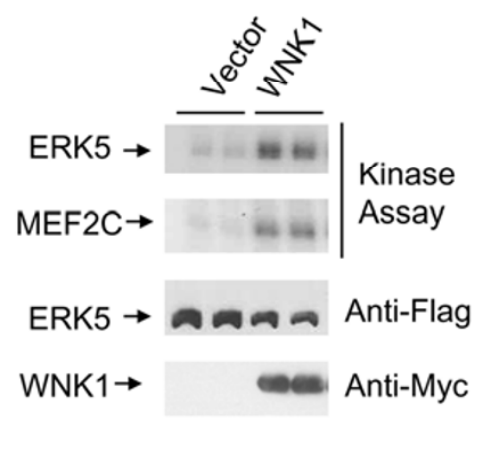

C

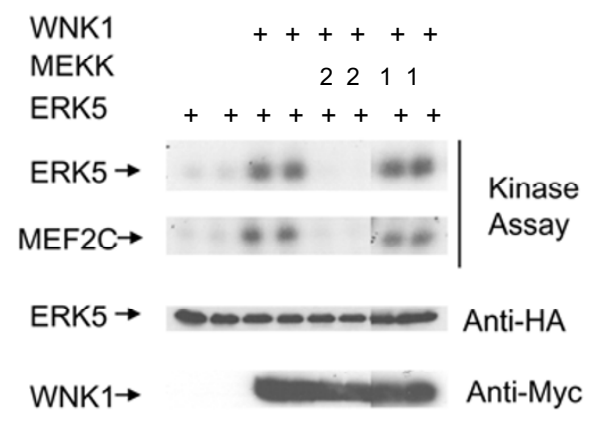

Fig. 3 WNK1 can activate the ERK5 MAPK pathway upstream of MEKK2 and MEKK3. (A) MAPK cascade: Left, the standard module; Right, an ERK5 module. (B) Activation of ERK5 by WNK1. (C) Effect of kinase-defective mutants of MEKK1and MEKK2 on activation of ERK5 by WNK1.

MAP2Ks directly, including MEK5 [15, 20]. We found that inactive mutants of MEKK2 or MEKK3, but not MEKK1 blocked ERK5 activation by WNK1 (Fig. 3C) [21], consistent with the cascade shown in Fig. 3A in which MEKK2 and MEKK3 are the MAP3Ks. Confirming the suggestion from these experiments, we also showed that WNK1 binds to, phosphorylates, and activates MEKK3. In vitro experiments showed that phosphorylation by WNK1 does not directly activate MEKK2 or MEKK3. Thus, the importance of phosphorylation of MEKK2 and MEKK3 by WNK1 remains uncertain. A number of proteins that function as MAP4Ks, in that they activate MAP3Ks, do not activate by phosphorylation. Several have been suggested to activate through protein-protein interactions, by directly inducing conformational changes, increasing oligomerization of the MAP3Ks, or localizing MAP3Ks to sites of activation. WNK1 may also be acting on MEKK2 and MEKK3 by one of these mechanisms.

Using RNAi we demonstrated that WNK1 is required for ERK 5 activation by low, physiological concentrations of EGF, as well as partially by $\mathrm{H}_{2} \mathrm{O}_{2}$ [21]. Although WNK1 is activated by osmotic stresses, it was not required for activation of ERK 5 by sorbitol or $\mathrm{NaCl}$. This may also suggest that the catalytic activity of WNK1 is not of major importance in the ERK5 pathway. Thus, regulators of the kinase activity are not necessarily the agents recruiting WNK1 to activate the ERK5 pathway. Partial clones of WNK1 and WNK2 were isolated from malignant prostate tissue and pancreatic adenocarcinoma, suggesting their involvement in cancer [22, 23]. The apparent importance of the ERK5 pathway in development along with the requirement for WNK1 in the activation of ERK 5 by EGF, may suggest that the WNK's linking to the ERK5 path- way may be the most important in normal and abnormal cell proliferation.

\section{A TWO-HYBRID SCREEN WITH THE WNK1 KINASE DOMAIN SUGGESTS A POTENTIAL MECHANISM TO REGULATE MEMBRANE FUNCTION}

Two-hybrid screens of a brain cDNA library with the kinase domain of WNK1 yielded synaptotagmin 2 [24]. Synaptotagmin 2 is a calcium sensor that facilitates fusion of vesicles with the plasma membrane [25]. The WNK1interacting portion of synaptotagmin 2 was its calciumbinding $\mathrm{C} 2$ domain. Synaptotagmin 2 and WNK1 bind as assessed by GST pull down assays, and by co-immunoprecipitation of endogenous proteins from cells. In contrast, WNK4 does not interact with synaptotagmin 2. Endogenous WNK1 and synaptotagmin 2 are found co-localized on a subset of secretory granules by immunofluorescence and sedimentation of homogenates from an insulin-secreting pancreatic $\beta$ cell line [24]. Phosphorylation of synaptotagmin 2 on threonine 202 can be detected by immunoblotting with a phospho-specific antibody and is enhanced by coexpression of WNK1. Phosphorylation by WNK1 increases the amount of $\mathrm{Ca}^{2+}$ required for synaptotagmin 2 binding to phospholipid vesicles and mutation of threonine 202 partially prevents this change (Fig. 4). Taken together, these findings suggest that phosphorylation of synaptotagmins by WNK1 is a mechanism to regulate $\mathrm{Ca}^{2+}$ sensing and the subsequent $\mathrm{Ca}^{2+}$-dependent interactions mediated by synaptotagmin $\mathrm{C} 2$ domains. The selectivity of WNK1 over WNK4 suggests that this may predominantly be a WNK1 function. These findings provide a biochemical mechanism that could lead to the retention or 


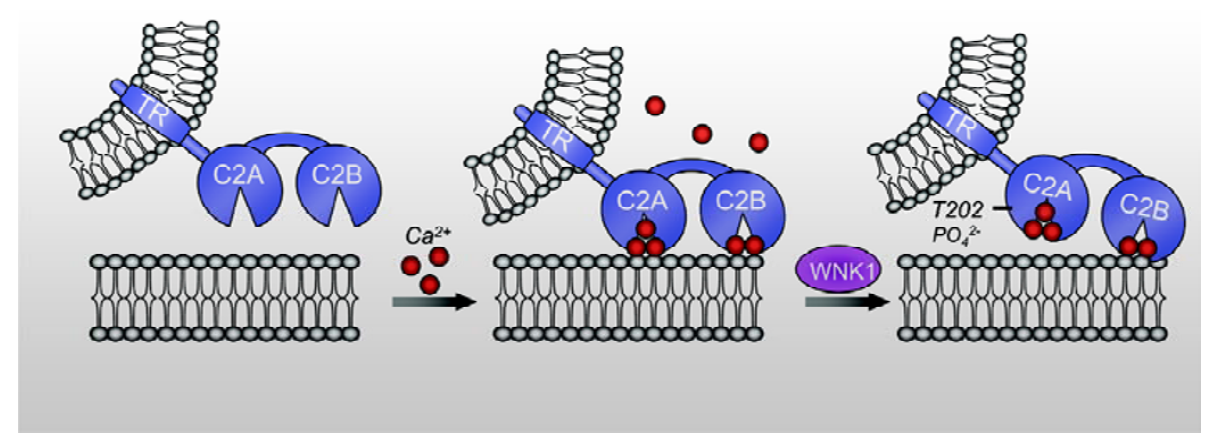

Fig. 4 Model showing the effect of WNK1 phosphorylation on the calcium-dependence of phospholipid binding by synaptotagmin 2 .

insertion of proteins in the plasma membrane. Because many ion transporters are regulated by membrane insertion or retrieval, WNK1 may be able to influence ion homeostasis through its effects on synaptotagmin function.

\section{WNKS-THE FUTURE}

The primary sequence and lack of identifiable domains in WNK proteins suggests that they function as scaffolds. WNK1, for example, has more than twenty PXXP motifs that are typical binding sites for SH3 domains. WNK4 has even more of these putative docking sites. The behavior of endogenous WNK1 in lysates is consistent with its forming a homo-tetramer and we have begun to identity sites of self-association. Given these properties, one possibility is that WNK hetero-oligomers may form under certain conditions. This would allow the functions of multiple WNK proteins to be acting as a single complex. It also seems likely that the interactions orchestrated by WNK proteins may be their most significant feature. It has been suggested that the average $50 \mathrm{kD}$ protein will interact with 9 other proteins, not including enzyme-substrate associations [26]. If this relationship can be extrapolated to proteins of large size, then one might predict that WNK1 will bind to as many as 50 other proteins. Thus, the capacity of WNK proteins to influence cell functions may be substantially greater than we presently realize. The fact that disruption of the $W N K 1$ gene caused lethality during embryonic development strongly suggests that blood pressure regulation is only one of the roles performed by this unusual group of protein kinases.

\section{ACKNOWLEDGMENTS}

The authors thank other members of the Cobb laboratory for critical comments about the manuscript, other members of the UT Southwestern community for helpful discussions, and Dionne Ware for administrative assistance.
Work from the authors' laboratories was supported by grant GM53032 from the National Institutes of Health and grants I1243 (to MHC) and I1128 (to EJG) from the Welch Foundation.

\section{REFERENCES}

1 Xu B, English JM, Wilsbacher JL, et al. WNK1, a novel mammalian serine/threonine protein kinase lacking the catalytic lysine in subdomain II. J Biol Chem 2000; 275:16795-801.

2 Verissimo F, Jordan P. WNK kinases, a novel protein kinase subfamily in multi-cellular organisms. Oncogene 2001; 20:5562-9.

3 Murakami-Kojima M, Nakamichi N, Yamashino T, Mizuno T. The APRR3 component of the clock-associated APRR1/TOC1 quintet is phosphorylated by a novel protein kinase belonging to the WNK family, the gene for which is also transcribed rhythmically in Arabidopsis thaliana. Plant Cell Physiol 2002; 43:67583.

4 Nakamichi N, Murakami-Kojima M, Sato E, et al. Compilation and characterization of a novel WNK family of protein kinases in Arabiodpsis thaliana with reference to circadian rhythms. Biosci Biotechnol Biochem 2002; 66:2429-36.

5 Morrison DK, Murakami MS, Cleghon V. Protein kinases and phosphatases in the Drosophila genome. J Cell Biol 2000; 150: F57-F62.

6 Wilson FH, Disse-Nicodeme S, Choate KA, et al. Human hypertension caused by mutations in WNK kinases. Science 2001; 293:1107-12.

7 Zambrowicz BP, Abuin A, Ramirez-Solis R, et al. Wnk1 kinase deficiency lowers blood pressure in mice: A gene-trap screen to identify potential targets for therapeutic intervention. Proc Natl Acad Sci U S A 2003; 100:14109-14.

8 Min X, Lee BH, Cobb MH, Goldsmith EJ. Crystal structure of the kinase domain of WNK1, a kinase that causes a hereditary form of hypertension. Structure 2004; 12:1303-11.

$9 \mathrm{Xu} \mathrm{B}$, Min X, Stippec S, et al. Regulation of WNK1 by an autoinhibitory domain and autophosphorylation. J Biol Chem 2002; 277:48456-62.

10 Chen Z, Gibson TB, Robinson F, et al. MAP kinases. Chem Rev 2001; 101:2449-76. 
11 Lewis TS, Shapiro PS, Ahn NG. Signal transduction through MAP kinase cascades. Adv Cancer Res 1998; 74:49-139.

12 Yan C, Luo H, Lee JD, Abe J, Berk BC. Molecular cloning of mouse ERK5/BMK1 splice variants and characterization of ERK5 functional domains. J Biol Chem 2001; 276:10870-8.

13 Widmann C, Gibson S, Jarpe MB, Johnson GL. Mitogen-activated protein kinase: conservation of a three-kinase module from yeast to human. Physiol Rev 1999; 79:143-80.

14 Pearson G, Robinson F, Beers GT, et al. Mitogen-activated protein (MAP) kinase pathways: regulation and physiological functions. Endocr Rev 2001; 22:153-83.

15 Chao TH, Hayashi M, Tapping RI, Kato Y, Lee JD. MEKK3 directly regulates MEK5 activity as part of the big mitogen- activated protein kinase 1 (BMK1) signaling pathway. J Biol Chem 1999; 274:36035-8.

16 Chayama K, Papst PJ, Garrington TP, et al. Role of MEKK2MEK5 in the regulation of TNF-alpha gene expression and MEKK2-MKK7 in the activation of c-Jun N-terminal kinase in mast cells. Proc Natl Acad Sci U S A 2001; 98:4599-604.

17 Kato Y, Kravchenko VV, Tapping RI, et al. BMK1/ERK5 regulates serum-induced early gene expression through transcription factor MEF2C. EMBO J 1997; 16:7054-66.

18 Kamakura S, Moriguchi T, Nishida E. Activation of the protein kinase ERK5/BMK1 by receptor tyrosine kinases. Identification and characterization of a signaling pathway to the nucleus. J Biol Chem 1999; 274:26563-71.
19 Watson FL, Heerssen HM, Bhattacharyya A, et al. Neurotrophins use the Erk5 pathway to mediate a retrograde survival response. Nat Neurosci 2001; 4:981-8.

20 Sun W, Kesavan K, Schaefer BC, et al. MEKK2 associates with the adapter protein Lad/RIBP and regulates the MEK5-BMK1/ ERK5 pathway. J Biol Chem 2001; 276:5093-100.

$21 \mathrm{Xu}$ B, Stippec S, Lenertz L, et al. WNK1 activates ERK5 by an MEKK2/3-dependent mechanism. J Biol Chem 2004; 279:782631.

22 Moore TM, Garg R, Johnson C, et al. PSK, a novel STE20-like kinase derived from prostatic carcinoma that activates the c-Jun $\mathrm{N}$-terminal kinase mitogen-activated protein kinase pathway and regulates actin cytoskeletal organization. J Biol Chem 2000; 275 : 4311-22.

23 Ito M, Shichijo S, Tsuda N, et al. Molecular basis of T cellmediated recognition of pancreatic cancer cells. Cancer Res 2001; 61:2038-46.

24 Lee BH, Min X, Heise CJ, et al. WNK1 phosphorylates synaptotagmin 2 and modulates its membrane binding. Mol Cell 2004; 15:741-51.

25 Geppert M, Goda Y, Hammer RE, et al. Synaptotagmin I: a major $\mathrm{Ca}^{2+}$ sensor for transmitter release at a central synapse. Cell 1994; 79:717-27.

26 Serebriiskii IG, Mitina O, Pugacheva EN, et al. Detection of peptides, proteins, and drugs that selectively interact with protein targets. Genome Res 2002; 12:1785-91. 